\title{
Observation on the Effect and Safety of Manipulation Combined with Taohong Siwu Decoction in the Treatment of Elbow Joint Adhesion after Fracture
}

\author{
Kun Ren, Guimei Wei, Yanjie Li, Ruoxing Xing, Hong Cheng \\ Henan Provincial Hospital of Traditional Chinese Medicine, Second Affiliated Hospital of Henan University \\ of Traditional Chinese Medicine, Zhengzhou, Henan, 450008, China
}

Keywords: Manipulation combined with taohong siwu decoction, Elbow joint adhesion after fracture, Effect

\begin{abstract}
Objective: To study the effect and safety of manipulation combined with Taohong Siwu Decoction in the treatment of elbow joint adhesion after fracture. Methods: In this study, patients with elbow joint adhesion after fracture were taken as the research object. 200 cases were admitted from March 2018 to March 2019. According to the principle of random drawing and grouping, they were divided into observation group 100 cases and control group 100 cases, respectively Manipulation combined with Taohong Siwu Decoction and conventional treatment methods were used to analyze and compare the indicators of the two groups. Results: The VAS score of the observation group was lower than that of the control group $(\mathrm{P}<0.05)$; the total effective rate of the observation group was higher than that of the control group $(\mathrm{P}<0.05)$; the incidence of adverse reactions in the observation group was lower than that of the control group $(\mathrm{P}<0.05)$. Conclusion: The treatment of elbow joint adhesion patients after fracture with manipulation combined with Taohong Siwu Decoction has achieved significant results.
\end{abstract}

\section{Introduction}

The incidence of elbow joint adhesions after fractures continues to increase, which is likely to affect the health of patients. It is very important to implement an effective treatment method [1]. Therefore, this study analyzes the treatment effect of the combination of the method and the Taohong Siwu Decoction, see Below.

\section{Materials and Methods}

\subsection{Information}

In this study, patients with elbow joint adhesion after fracture were taken as the research object. 200 cases were admitted from March 2018 to March 2019. According to the principle of random drawing and grouping, they were divided into observation group 100 cases and control group 100 cases, respectively. Combined with Taohong Siwu Decoction and conventional treatment methods, the inclusion criteria: (1)Diagnosed as elbow joint adhesion after imaging diagnosis, (2)The patient is accompanied by varying degrees of limited joint movement and joint pain. Exclusion criteria: (1) Severe medical diseases, (2Drug allergy history. Observation group: 60 males and 40 females; the age range is $40-80$ years old, and the average age of 100 patients is $(60.21 \pm 1.27)$ years. Control group: 61 males and 39 females; the age range is 41-80 years old, and the average age of 100 patients is $(60.11 \pm 1.23)$ years. There was no significant difference in basic data between the two groups, $\mathrm{P}>0.05$.

\subsection{Method}

Implementation of the control group: conventional treatment.

After admission, both groups of patients underwent fracture fixation, anti-infection treatment, early functional rehabilitation training, and instructed patients to maintain a sitting position and relax. The operator should use hands to massage the elbow joint of the patient to prevent injury. For 
the best strength, the operator uses the thumb of the thumb to beat the patient's triceps, biceps, radial collateral ligament, and supinator muscles. Pay attention to maintaining a moderate intensity, so as to achieve the purpose of loosening adhesions and eliminating stasis. The operator uses the left hand to support the humerus of the affected limb, and uses the hand to hold the affected limb and the wrist to receive outwards. The movement range should be small to large, and the regularity of the technique should be ensured to prevent the patient from causing damage to the body. , From top to bottom, gently tap the patient's affected limb and rub it to achieve contact fatigue and local spasm [2].

Implementation of observation group: Manipulation combined with Taohong Siwu Decoction Modified and Subtracted Treatment.

On the basis of the control group, the modified Taohong Siwu Decoction was used: Codonopsis 15g, Safflower 6g, Atractylodes 15g, Fructus Aurantii 15g, Peach kernel 15g, Chuanxiong 15g, Rehmannia glutinosa 15g, Moutan bark 10g, Poria cocos 10g, Moxibustion licorice 10g, Salvia 10g, Angelica $10 \mathrm{~g}$, if the patient has symptoms of phlegm, add platycodon, almonds, etc., if the patient has symptoms of dry mouth and tongue, add raw rehmannia, adenophora, etc., take twice a day, at the same time The patients were treated with traditional Chinese medicines for fumigation and washing, including borneol, green wood incense, radix sylvestris, raw Chuanwu, safflower, chinensis, tongucao, salvia, etc., 30 minutes a day, three times a day, and two weeks of treatment [3].

\subsection{Observation Indicators}

Comparison of VAS score, incidence of adverse reactions and total effective rate between the two groups.

\subsection{Statistical Processing}

SPSS27.0 statistical software implements the analysis, and analyzes the research data ( $\mathrm{T}$ test method, Chi-square test method), and the result data $\mathrm{P}<0.05$ represents statistical significance.

\section{Results}

\subsection{Compare Vas Score}

The VAS score of the observation group was lower than that of the control group $(\mathrm{P}<0.05)$, as shown in Table 1.

Table 1: Analysis of Vas Score Indicators

\begin{tabular}{|l|l|l|}
\hline Gr & Case(n) & VAS \\
\hline Observation Gr & 100 & $1.21 \pm 0.31$ \\
\hline Control Gr & 100 & $2.61 \pm 1.27$ \\
\hline $\mathrm{t}$ & - & 6.4517 \\
\hline $\mathrm{P}$ & - & 0.0000 \\
\hline
\end{tabular}

\subsection{Comparison of Total Effective Rate}

The total effective rate of the observation group is higher than that of the control group $(\mathrm{P}<0.05)$, as shown in Table 2.

Table 2: Analysis of the Total Effective Rate Index

\begin{tabular}{|l|l|l|l|l|l|l|l|l|}
\hline Gr & Case(n) & $\begin{array}{l}\text { Markedly } \\
\text { effective(n) }\end{array}$ & \multicolumn{2}{l|}{ effective(n) } & \multicolumn{2}{l|}{ invalid(n) } & \multicolumn{2}{l|}{ Total effective rate(n;\%) } \\
\hline Observation Gr & 100 & 90 & 6 & 4 & & 96.00 \\
\hline Control Gr & 100 & 70 & 10 & 20 & & \\
\hline $\begin{array}{l}\text { Chi- } \\
\text { square } \\
\text { value }\end{array}$ & - & & & & & & \\
\hline P & - & & & & & & 0.0000 \\
\hline
\end{tabular}




\subsection{Compare the Incidence of Adverse Reactions}

The incidence of adverse reactions in the observation group was lower than that in the control group $(\mathrm{P}<0.05)$, as shown in Table 3 .

Table 3: Analysis Of the Indicators of the Incidence of Adverse Reactions

\begin{tabular}{|l|l|l|l|l|}
\hline Gr & Case(n) & Y(n) & N(n) & NG Rate \\
\hline Observation Gr & 100 & 4 & 96 & 4.00 \\
\hline Control Gr & 100 & 11 & 89 & 11.00 \\
\hline Chi-square value & - & & & 7.2613 \\
\hline P & - & & & 0.0127 \\
\hline
\end{tabular}

\section{Discussion}

According to related studies, elbow joint fracture is a very common type of fracture in the clinic, and it is also a clinically frequently-occurring and common disease. The patient is generally caused by violence and can also be caused by accidental surgical loss [4-7], patients It is prone to elbow joint adhesion and spasm or basic motor dysfunction, which seriously affects the patient's quality of life and also affects daily life. In Chinese medicine in my country, it is believed that the loss and prolonged immobilization after a fracture can easily lead to blockage of veins and stagnation of blood and blood. As time goes by, the invasion of wind, cold and dampness will lead to paralysis of the muscles and bones, and then adhesions are formed. . Clinically, it is believed that for people with elbow joint adhesion after fracture, the methods should be used to remove blood stasis and dredge collaterals, replenish qi and promote blood circulation. In traditional Chinese medicine treatment, the key to removing blood stasis and activating blood and dredging the channels and collaterals, so as to improve local microcirculation. To achieve the purpose of preventing loosening and adhesion [8-10].

Taohong Siwu Decoction is a common clinical traditional Chinese medicine decoction, which is mainly composed of a variety of traditional Chinese medicines, such as angelica, safflower, white peony root, rehmannia, chuanxiong, peach kernel, etc., with the purpose of dispelling blood stasis and dredging collaterals, promoting blood circulation and relieving pain, etc. It is widely used in the treatment of clinical diseases [11]. In this study, Taohong Siwu Decoction was used in patients with elbow joint adhesion after fracture. It was found to have immunomodulatory and anti-infective effects, and at the same time, it can improve the blood supply of the affected area, thereby reducing complications caused by blood stasis. In this study, all indicators of the observation group were better than those of the control group. The main reason was that safflower and peach kernels can dredge collaterals and remove blood stasis, angelica can nourish blood and promote blood circulation; while white peony can nourish blood and relieve pain; Poria has the power of soothing the liver and regulating qi; moxibustion and licorice as a reconciling medicine can reconcile various medicines, and play the power of removing blood stasis and dredging collaterals, replenishing qi and activating blood [12]. By applying the traditional Chinese medicine fumigation and washing method on the basis of the above treatment, a very significant effect can be produced, mainly through the use of hot fumigation to deliver the medicine into the skin tissue of the patient, which is beneficial to the acceleration of the local blood circulation of the human body, thereby achieving the elimination of congestion, The purpose of reducing swelling has the effects of removing blood stasis and dredging collaterals, warming menstruation and promoting blood circulation, and can enhance the efficacy of drugs and drugs, improve the prognosis of patients, and improve the quality of life of patients [13].

Studies have shown that the VAS score of the observation group is lower than that of the control group $(\mathrm{P}<0.05)$; the total effective rate of the observation group is higher than that of the control group $(\mathrm{P}<0.05)$; the incidence of adverse reactions in the observation group is lower than that of the control group $(\mathrm{P}<0.05)$.

To sum up, through the implementation of manipulation combined with Taohong Siwu Decoction for patients with elbow joint adhesion after fracture, significant results have been 
achieved, and it is worthy of promotion and application in clinical practice.

\section{References}

[1] Zang Penglei. Analysis of the curative effect of manipulation combined with Taohong Siwu Decoction in the treatment of elbow joint adhesions after fracture. The world's latest medical information abstracts (continuous electronic journals), 2019,19(98):228-229.

[2] Zhong Linkuan, Li Wu, Mao Fei, et al. Clinical observation on treatment of elbow joint adhesion after fracture with manipulation combined with Taohong Siwu Decoction. Frontier of Medicine, 2018, 8(13): 328.

[3] Yu Fang, Gao Hongyi, Fu Xiali, et al. Clinical observation on the treatment of elbow joint adhesions after fracture with manipulation combined with Taohong Siwu Decoction. Shaanxi Journal of Traditional Chinese Medicine, 2017, 38(8): 1037-1038.

[4] Meng Xianyun. Clinical observation of combined manipulation and Taohong Siwu Decoction (addition and subtraction) for the treatment of elbow joint adhesion after fracture. Electronic Journal of Clinical Medicine, 2018, 5(22): 169.

[5] Wang Xiangyang, Tian Aiping. Clinical observation on 41 cases of elbow adhesion after fracture treated with manipulation combined with Taohong Siwu Decoction. New Chinese Medicine, 2016, 48(3): 101-103.

[6] Wang Dan, Zhao Huimei, Tang Yu. The effect of fumigation and washing with traditional Chinese medicine on the improvement of joint mobility after elbow fracture recovery and nursing experience. Shanxi Medical Journal, 2019, 48(4): 512-514.

[7] Cosman F, Crittenden DB, Ferrari S, et al. FRAME Study: The Foundation Effect of Building Bone With 1 Year of Romosozumab Leads to Continuf Romosozumab Lea Fracture Risk After Transition to Denosumab. Journal of Bone \& Mineral Research, 2018, 21(2):178-186.

[8] Liu Chaosheng, Long Xianliang, Yang Mingxia, et al. System rehabilitation after elbow fracture surgery. Massage and Rehabilitation Medicine, 2017, 8(4): 13-15.

[9] Govaert GAM, Bosch P, Ijpma FFA, et al. High diagnostic accuracy of white blood cell ell scintigraphy for fracture related infections for fracture related infections: Results of a large retrospective ell scintigraphyell scintigraphy for fracture related infection for fracture related infectionsingle-center study. Injury-international Journal of the Care of the Injured,2019, v.8(04):110-121.

[10] Zhang Mingchao. The effect of rehabilitation exercise combined with nutritional intervention on the rehabilitation of lower humeral fractures after internal fixation. Journal of Hainan Medical College, 2016, 22(18): 2199-2201.

[11] Du Hongxia. Rehabilitation treatment of 50 patients with elbow joint fractures after operation. China Modern Medicine Application, 2015, 9(13): 268-270.

[12] Li Xiufang, Guo Yingjun, Wang Liping, et al. The effect of early functional training on the prognosis of patients with elbow joint fractures. Journal of Hainan Medical College, 2014, 20(8): 1151-1153.

[13] Felicia C, Crittenden DB, Serge F, et al. FRAME Study: The Foundation Effect of Building Boneell scintigraphy for fracture related infection with 1 Year of Romosozumab Leads to Continued Lower Fracture Risk After Transition to Denosumab. Journal of bone and mineral research: the official journal of the American Society for Bone and Mineral Research, 2018, 41(3):581-596. 